\title{
Zinc Gluconate and the Common Cold: a Controlled Clinical Study
}

\author{
JC GODFREY ${ }^{l}$, B CONANT SLOANE ${ }^{2}$, D S SMITH ${ }^{2}$, J H TURCO ${ }^{2}$, \\ NMERCER ${ }^{2}$ AND NJ GODFREY ${ }^{l}$
}

${ }^{1}$ Huntingdon Valley, Pennsylvania, USA; ${ }^{2}$ Dartmouth College Health Service, Hanover, New Hampshire, USA

A report in 1984 on the success of zinc gluconate against common cold symptoms could not be confirmed in three subsequent studies, which are now known to have used formulations that inactivated zinc. A non-chelating formulation including glycine, which releases $93 \%$ of contained zinc into saliva, was tested in a randomized, placebo-controlled, double-blind trial in 73 young adults. Efficacy was recorded in symptom diaries using a symptom severity rating. Patients' symptoms first appeared 1.34 days prior to entry to the study in both groups. Disappearance of symptoms occurred after an additional 4.9 days for zinctreated patients versus 6.1 days for placebo-treated patients. A difference was noted in the efficacy of treatment if it was started 1 day after symptom onset: cold duration was an additional 4.3 days in zinc-treated patients compared with 9.2 days for placebo-treated patients. Cough, nasal drainage and congestion were the symptoms most affected, and only mild side-effects were noted.

KEY WORDS: ZINC GLUCONATE - GLYCINE; UPPER RESPIRATORY TRACT INFECTION; COLD SYMPTOM RELIEF 


\section{INTRODUCTION}

Attempts made to duplicate the success which Eby et al. ${ }^{1}$ had in 1984 in reducing the duration of the common cold using zinc gluconate have generally been disappointing ${ }^{2}$, the probable reason being that zinc gluconate was inactivated by additives used to mask its unpleasant taste. It has been demonstrated that of these agents, e.g. citric acidtartaric acid, ${ }^{3}$ or mannitol/sorbitol,inactivate zinc by chelation in salivá. Unflavoured zinc gluconate and the zinc gluconate - glycine (ZGG) lozenges used in the present study release $90-93 \%$ of zinc ion 6 syvhereas citric acid $^{2}$ and mannitol/sorbitôl formulations release no zinc ions when dissolved in the consent.

mouth. If the presence of zinc ions in the mouth is required for an effect on the common TREATMENT

cold, chelation of zinc may be the reason why A total of four candidate placebos containing the subsequent studies were unsuccessful. different ratios of highly astringent tannic This is further suggested by another study, acid and traces of saccharin were investigated, which found a significant reduction of and a taste test study was conducted to symptoms when a non-chelating formulation determine which of these formulations most was used?

The present study was carried out to test the hypothesis that pleasant-tasting ZGG lozenges that release $93 \%$ of the ionic zinc into saliva may produce similar efficacy to that originally reported by Eby al. ${ }^{1}$

\section{P ATIENTS AND METHODS}

\section{PATIENTS}

cough; fever; headache; hoarseness; muscle ache; nasal drainage; nasal congestion; scratchy throat; sore throat; and/or sneezing. Participants were also identified as having had the symptoms for either 1 day or 2 days prior to entering the study. Participants were excluded if they had a positive bacteriological throat culture, were pregnant, or had symptoms consistent with influenza or any other illness. The study was approved by The Committee for the Protection of Human Patients of the Dartmouth Medical Administration, and prior to entering the trial all patients gave their written informed closely matched the ZGG lozenges. Unrelated symptom-free adult volunteers (four men, four women) were asked to compare each of the four placebos with active treatment lozenges. A Latin-square design was used to ensure that the placebo was presented in the first half of the time for each placebo and that each placebo was tested in each of the four trial positions twice. The placebo that subjects considered most like ZGG in astringency and pleasantness was selected; it contained US Pharmacopoeia tannic acid, glycine and

A total of 87 patients were recruited from calcium saccharinate in an orange-flavoured, among Dartmouth College students and staff boiled candy base, weighed $4.5 \mathrm{~g}$ and was who spontaneously presented to the cold identical to the ZGG lozenges in all clinic at the Dartmouth College Health Service characteristics. The ZGG lozenges, which were over a 28-day period. All patients were prepared in the same boiled candy base as the examined and diagnosed by a physician or placebo contained glycine and zinc gluconate nurse clinician as having shown, for no more trihydrate, and the zinc content was $5.26 \pm$ than 2 days, between two and nine symptoms $0.20 \mathrm{mg} / \mathrm{g}$, or $23.7 \mathrm{mg}$ zinc in each $4.5 \mathrm{~g}$ consistent with a common cold. These symptoms included any of the following: 
lozenge. Placebo and ZGG lozenges were bacteriologically sterile.

Patients were provided with 16 lozenges at their first visit to the clinic (day 0) and at the second visit, 2 days later, a further 64 lozenges were prescribed if symptoms persisted. They were instructed (both verbally and in writing) to suck, not chew, the lozenges as required but at not less than 2-h intervals taking up to a maximum of eight lozenges per day. Patients were also provided with paracetamol and instructed not to exceed the dosage stated on the label, nor to use any other form of medication.

\section{STUDY DESIGN}

Randomization by a third party was used to assign the 87 participants to treatment groups. A pharmacist, using a randomization table provided by the study statistician, packaged containers for individual subjects with lozenges according to the production run number and subject identification number. Patients, investigators and the pharmacist were, therefore, all blinded as to which treatment individual patients had received.

All patients kept diaries recording the severity of their symptoms upon enrollment, at 6 and $12 \mathrm{~h}$ after the first dose of the study medication, and at $20.00 \mathrm{~h}$ on each subsequent day. They were instructed to rate the severity of 10 cold symptoms on a scale of $0-3(0$, none; 1 , mild; 2 , moderate; and 3, severe) and, in addition, were asked to record any sideeffects.

The containers issued by the investigators were returned with unused lozenges so that counts could be made and daily usage by the two treatment groups could be compared.

\section{STATISTICAL ANAL YSIS}

Comparisons of the demographic data for the placebo- and ZGG-treated groups, as well as a determination of the patient's level of awareness as to which treatment they were receiving were performed using the $\chi^{2}$-test. Student's $t$-test was used to compare average daily usage of lozenges by the two treatment groups and a two-way analysis of variance was used to test treatment effect and immediacy of treatment. The level of statistical significance adopted for all comparisons was $5 \%$.

\section{RESULTS}

A total of eight ZGG- and six placebo-treated patients withdrew from the trial leaving 35 and 38 evaluable patients, respectively. Illnesses that resulted in patients withdrawing from the study were as follows: two patients had bronchitis and one had viral gastroenteritis in the placebo treatment group; and there was one patient with influenza and one with a bacterial infection in the ZGG treatment group. Other reasons for withdrawing were: failure to appear at follow-up (three ZGG- and one placebo-treated patient); efficacy doubted by the patient (one ZGG- and one placebotreated patient); nausea (one ZGG- and one placebo-treated patient); and sports injury (one ZGG-treated patient).

Demographic data for the 73 patients evaluated in the trial are shown in Table 1. The age range for the ZGG treatment group (18 -40 years) was greater than that of patients in the placebo group (18 - 24 years); however, the mean duration of colds for those patients in the ZGG treatment group who were older than 24 years was the same as for those who were under 25 years of age. The mean number of days that the patients had experienced symptoms prior to entering the programme was 1.34 days, the same mean for both groups. There was no significant $(P<0.05)$ difference between the ZGG and placebo treatment groups as to the distribution of female and male participants. The ethnic origin of the participants was as follows: 59 Caucasians; 
Demographic data for 73 patients with common cold symptoms treated with up to eight placebo or zinc gluconate - glycine (ZGG) lozenges per day for 7 days

\begin{tabular}{lcc}
\hline & \multicolumn{2}{c}{ Treatment group } \\
\cline { 2 - 3 } Characteristic & ZGG & Placebo \\
\hline No. of patients & 35 & 38 \\
Male & 23 & 21 \\
Female & 12 & 17 \\
Age (years) & & \\
Median & 21.2 & 20.1 \\
Range & $18-40$ & $18-24$ \\
Mean no. days & & \\
with symptoms on entry & 1.34 & 1.34 \\
\hline
\end{tabular}

six Asians; four blacks; one native American; and three of unknown origin.

\section{EFFICACY}

Mean numbers of symptoms at entry were 6.5 \pm 1.6 in ZGG-treated patients and $6.6 \pm 1.6$ in placebo-treated patients. The size of the reduction in both frequency and severity of individual symptoms after 7 days of treatment is presented in Table 2. The difference between ZGG and placebo, by the criterion of symptom severity reduction, was noticable by day 5 and was significant $(P<0.025)$ by day 7 . At day 7 , five $(14.3 \%)$ of the $35 \mathrm{ZGG}$-treated patients had a total of 15 symptoms, whereas 17 (44.7\%) of the 38 placebo-treated patients had a total of 45 symptoms.

In the present study, the strict criterion of complete disappearance of all symptoms was used as the definition of the cold being over. Considering all treated patients, the average duration of the cold after treatment was initiated was 1.27 days less $(t=2.01, P<0.05)$ for the ZGG treatment group (4.86 days) than for the placebo group (6.13 days). After day 4, the rate at which patients taking ZGG became symptom-free increased rapidly compared with the placebo-treated patients and became significantly $(P=0.05)$ different compared with placebo by day 6 (Table 2 ).

Anecdotal evidence suggested that the earlier ZGG treatment was initiated the shorter was the duration of the cold. It was planned a priori to test the effect of day of entry on duration of the cold after entering study. The 73 evaluable patients consisted of 21 ZGGand 23 placebo-treated patients who had had symptoms for 1 calendar day prior to entering (day 1 patients), and 14 ZGG- and 15 placebotreated patients with symptoms for 2 calendar days prior to entry (day 2 patients). In a twoway analysis of variance both the treatment effect (zinc versus placebo) and the number of days with symptoms prior to treatment (day 1 
Frequency and severity of cold symptoms remaining in 73 patients with common cold symptoms after treatment with up to eight placebo or zinc gluconate-glycine (ZGG) lozenges per day for 7 days

\begin{tabular}{|c|c|c|c|c|}
\hline \multirow{3}{*}{$\frac{\text { Symptom }}{\text { Cough }}$} & \multicolumn{2}{|c|}{ Frequency ${ }^{a}$} & \multicolumn{2}{|c|}{ Severity scoreb } \\
\hline & ZGG & \multirow{2}{*}{$\frac{\text { Placebo }}{7 / 31(22.6 \%)}$} & \multirow{2}{*}{$\frac{\text { ZGG }}{2 / 44(4.5 \%)}$} & Placebo \\
\hline & $2 / 26 \quad(7.7 \%)$ & & & $8 / 48(16.7 \%)$ \\
\hline Nasal drainage & $4 / 31(12.9 \%)$ & $11 / 32(34.4 \%)$ & $5 / 59(8.5 \%)$ & $12 / 61(19.7 \%)$ \\
\hline Nasal congestion & $3 / 24(12.5 \%)$ & $12 / 28(42.9 \%)$ & $3 / 41(7.3 \%)$ & $14 / 48(29.2 \%)$ \\
\hline Hoarseness & $1 / 27 \quad(3.7 \%)$ & $3 / 27(11.1 \%)$ & $1 / 46(2.2 \%)$ & $3 / 40 \quad(7.5 \%)$ \\
\hline Sore throat & $2 / 29 \quad(6.9 \%)$ & $4 / 28(14.3 \%)$ & $2 / 51(3.9 \%)$ & $6 / 44(13.7 \%)$ \\
\hline Scratchy throat & $0 / 23 \quad(0 \%)$ & $2 / 26 \quad(7.7 \%)$ & $0 / 36 \quad(0 \%)$ & $2 / 39 \quad(5.1 \%)$ \\
\hline Sneezing & $1 / 21 \quad(4.8 \%)$ & $3 / 28(10.7 \%)$ & $1 / 32(3.1 \%)$ & $3 / 44 \quad(6.8 \%)$ \\
\hline Headache & $2 / 22 \quad(9.1 \%)$ & $2 / 28 \quad(7.1 \%)$ & $2 / 37(5.4 \%)$ & $3 / 48 \quad(6.3 \%)$ \\
\hline Muscle ache & $1 / 16 \quad(6.3 \%)$ & $1 / 15 \quad(6.7 \%)$ & $1 / 21(4.8 \%)$ & $1 / 24 \quad(4.2 \%)$ \\
\hline
\end{tabular}

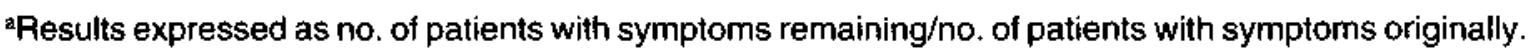

'Scored on a four-point scale (0, none; 3 , severe).

and versus day 2) were significant at the 0.05 level; there was no interaction effect.

For ZGG-treated patients, the number of days with symptoms while in the study for those who had had symptoms for 1 day before treatment was 4.29 days compared with 5.71 days for those who had had 2 days of symptoms before treatment (Fig.1); this difference of 1.42 days was significant $(t=-2.198, P=$ $0.035)$. When the duration of the cold prior to beginning treatment was allowed for, the significance of the differences between the day 1 and the day 2 ZGG treatment groups was increased to $P<0.001(t=-3.737)$. In terms of severity of symptoms following $\mathrm{ZGG}$ treatment, patients in the day 1 treatment group had less than $5 \%$ of their original severity in three symptoms compared with six remaining symptoms with as much as
$16.7 \%$ of the original severity among the day 2 ZGG-treated patients (Fig. 2). The analysis by day of entry (ZGG versus placebo) indicated that by day 7 there was only one day 1 ZGGtreated patient with a symptom severity score of 1 in each of three symptoms, compared to eight day 1 placebo-treated patients with an average severity score of 2.6 in an average of 2.4 symptoms. Drainage and congestion still had incidences of $33 \%$ and $31 \%$, respectively, among placebo-treated patients, but the ZGG-treated patient reported only a 5\% residual drainage incidence and recorded no congestion at all (Fig. 3).

\section{EFFECT OF PLACEBO}

For a truly inactive placebo, the total duration of symptoms would be expected to be the same. i.e. those who had entered after 1 day of 

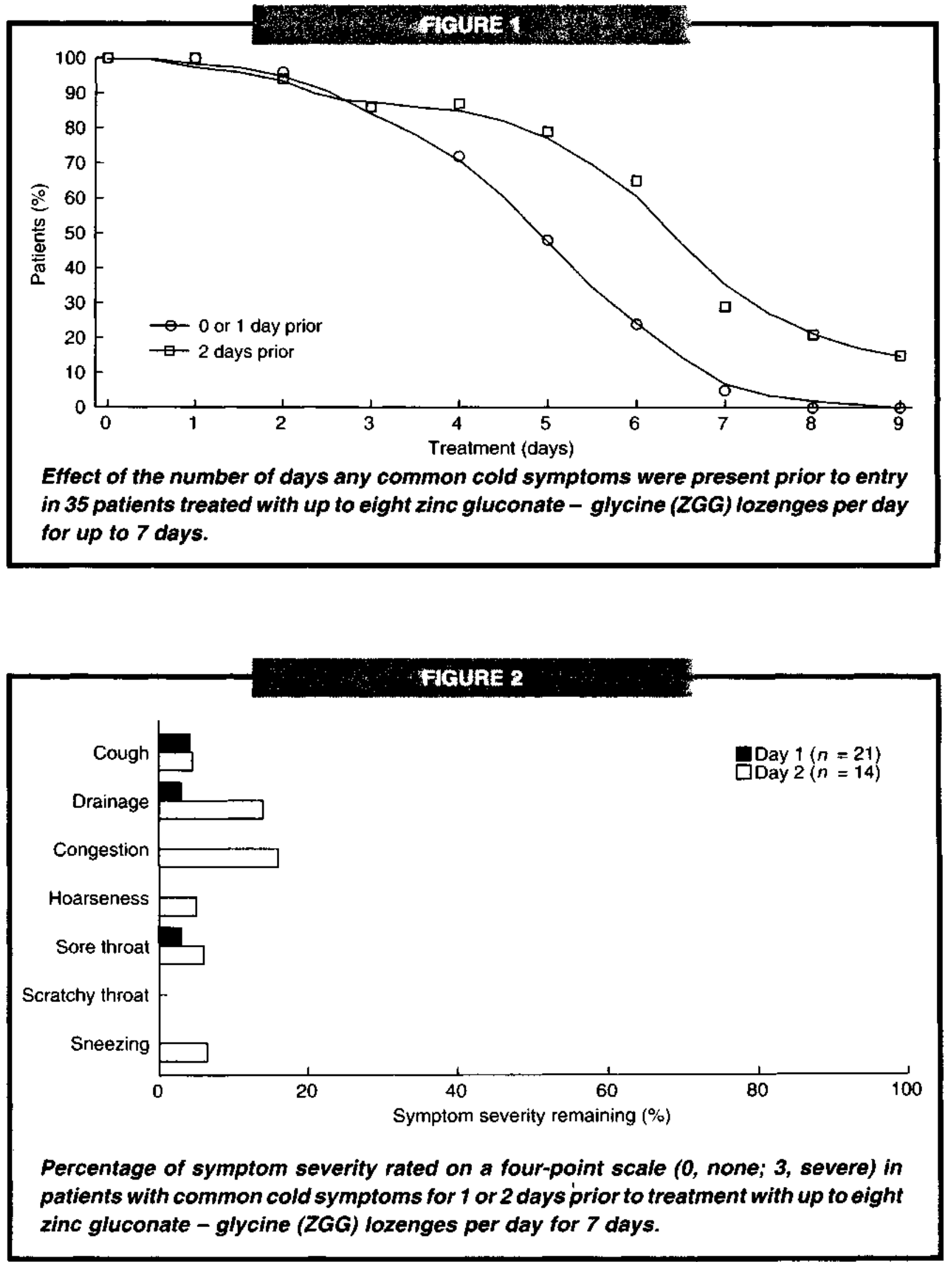


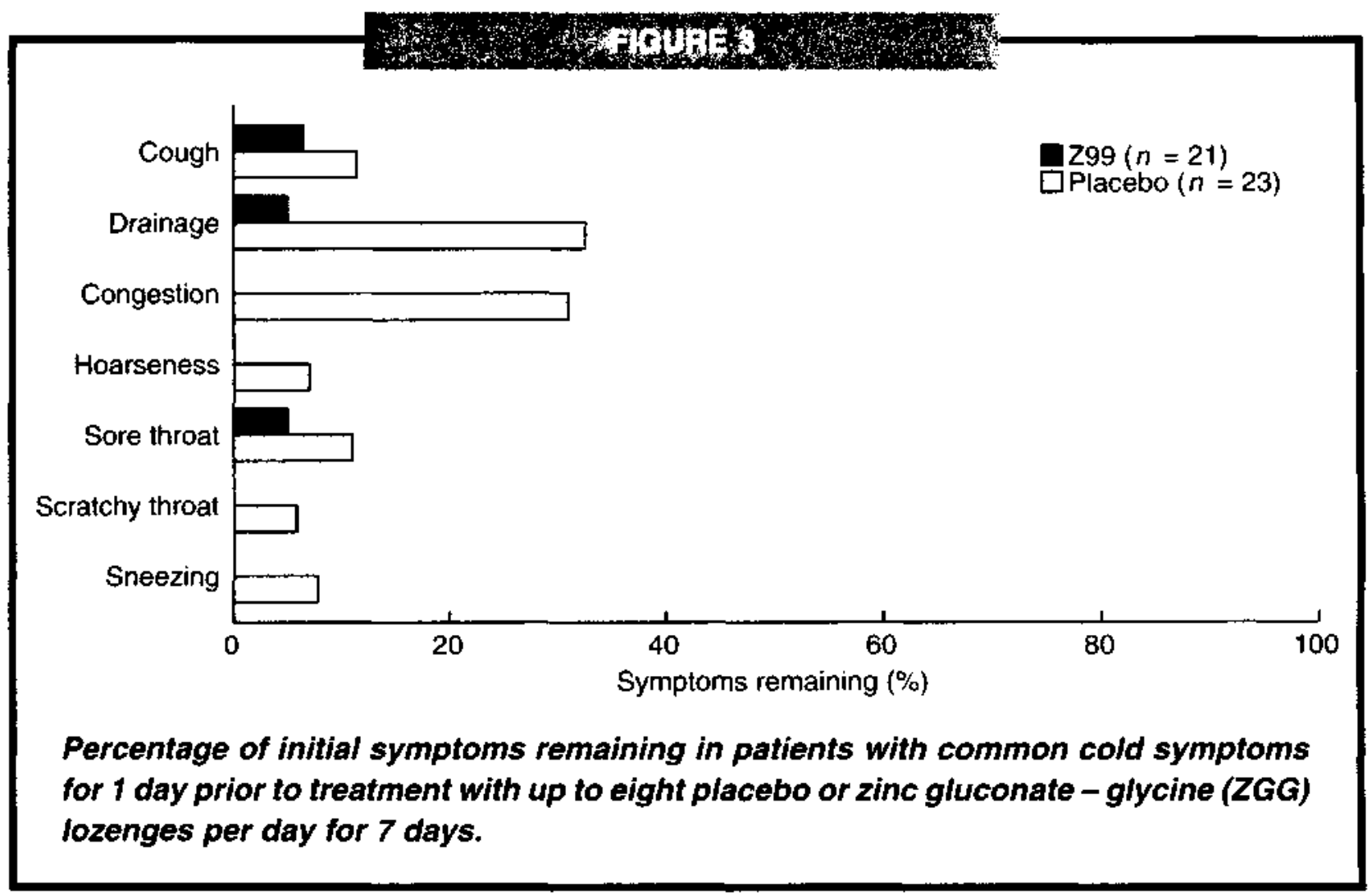

symptoms would be expected to be symptomatic, and thus receiving treatment, for 1 day longer than if they had entered having had symptoms for 2 days prior to entry. In the present study, those patients on placebo who entered with 1 day, or less, of symptoms were symptomatic for a total of 2.6 days less than those who had had symptoms for 2 days prior to entry. The $t$-statistic for the net difference in favour of the day 1 placebo treatment group was computed to be -2.954 , $(P<0.01)$. The implication is that the placebo used in the study was not totally inactive and that it seems to have produced some benefit if treatment was started early in the course of illness.

The 9.1-day duration of symptoms in the patients receiving placebo who entered after having had symptoms for 2 days more closely approximates the duration expected of a true placebo. Compared with the ZGG-treated patients who started with 1 day of symptoms and had symptoms for a total of 5.3 days, the true placebo-treated patients had symptoms 1.7 times as long.

Looking at the 'day of entry' effect in Figs 1 and 4, it can be observed how much better patients treated with ZGG on day 1 fared than did those who first received ZGG on day 2 . Charted in Fig. 5 is the large difference on day 7 favouring patients treated with ZGG on day 1 over patients treated with placebo on day 1 : one ZGG-treated patient had three symptoms remaining, whereas eight placebo-treated patients had an average of 2.75 symptoms. The day 2 patients did not fare as well (Fig. 6): four day 2 ZGG-treated patients had a total of 12 symptoms compared with nine day 2 placebo-treated -patients with 23 symptoms remaining. Compared with day 1 patients, day 2 patients benefitted relatively moderately from the ZGG treatment. 

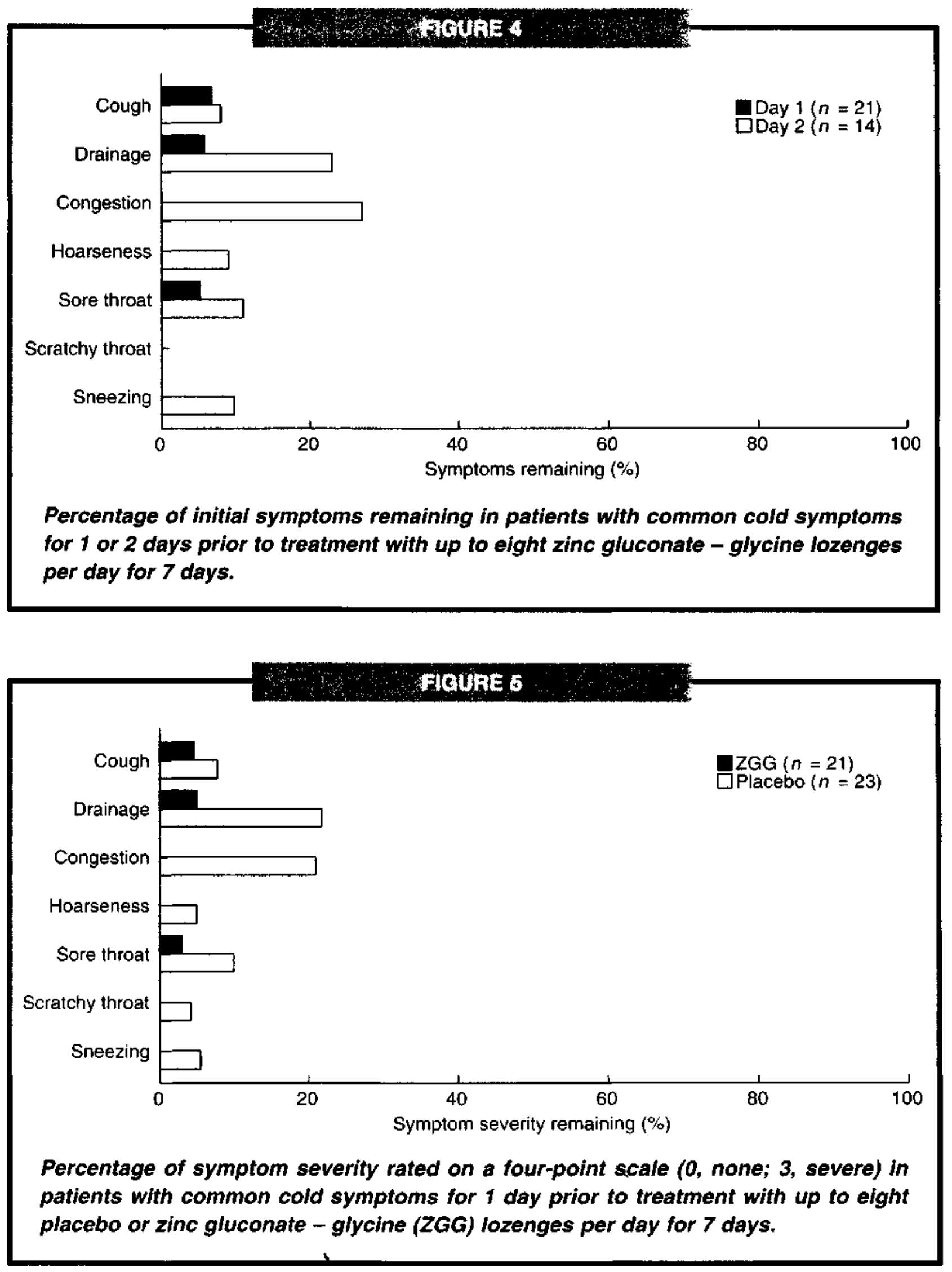


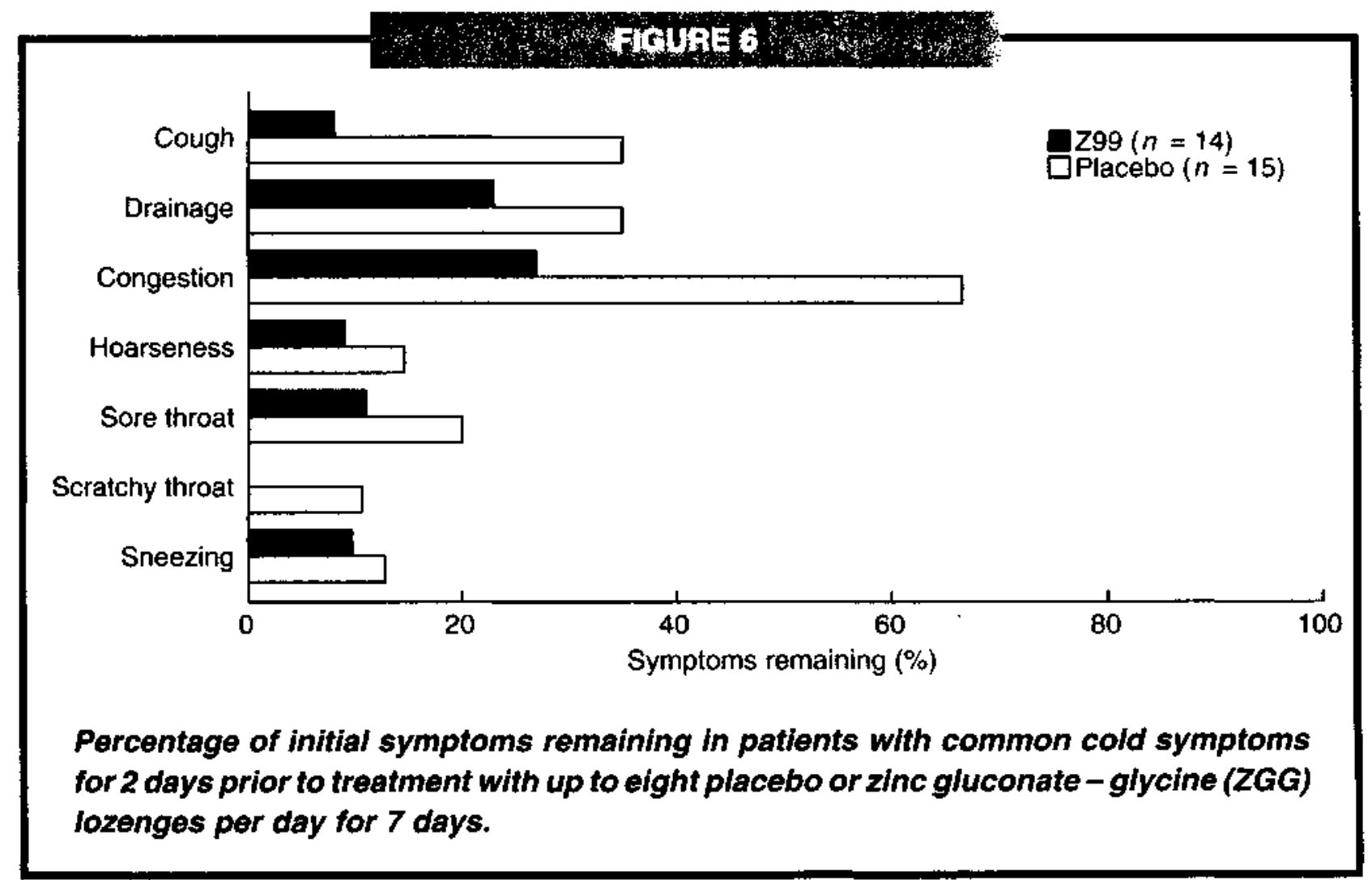

\section{PATIENTS' INTERPRETATION OF TREATMENT}

At their final visits, the patients were asked by the nurse which treatment they thought they had received. A total of 19 patients in each group guessed correctly; 12 of those who had received ZGG and 15 of those who received the placebo guessed incorrectly. In each treatment group there were four who did not know, and these eight patients were divided evenly among the four cells for the test of independence. The resulting $\chi^{2}$-value of 0.8975 was not significant; thus, the patients did not know which treatment they had received.

\section{MEDICATIONS TAKEN}

Returned medication counts showed a high degree of adherence to protocol in both the ZGG and placebo treatment groups. Of the expected daily use of eight lozenges, placebotreated patients used $7.1 \pm 1.4$ lozenges per day of participation and ZGG-treated patients used $8.1 \pm 1.7$ lozenges per day; the difference in usage was not significant. Paracetamol consumption was low at 7.9 tablets per patient in the 20 placebo-treated patients and 5.9 tablets per patient in the 20 ZGG-treated patients who used the analgesic.

\section{ADVERSE EFFECTS}

Of the placebo-treated patients, $30 \%$ had adverse experiences that were considered to be related in some degree to the treatment with study medications compared with $35 \%$ of the patients who received ZGG. The most common adverse experience in both treatment groups was gastro-intestinal discomfort, which occurred on 12 occasions in placebo-treated patients and 13 times in ZGG-treated patients. 
The only other common complaints were mouth irritation (including taste aberrations), which occurred eight times in placebo-treated patients and 12 times in ZGG-treated patients; dizziness, three times in ZGG-treated patients; and headache once in a ZGG-treated patient. Placebo patients reported one instance each of 'shakes', blood in mucus, weakness, drowsiness and skin discoloration. A total of 29 adverse experiences were recorded in 20 ZGG-treated patients and a total of 25 in 15 placebo-treated patients, or 1.5 and 1.7 per patient, respectively. All adverse experiences resolved spontaneously without further treatment.

\section{DISCUSSION}

It was hypothesized a priori that zinc may have a direct antiviral activity in the oral cavity and that its astringency may be acting upon the trigeminal and/or other nerves that are known to innervate both the oral and the nasal cavities, thus acting to suppress or reduce symptoms. Suppression of symptoms may render the upper respiratory tract a less favourable environment for viral replication. It has been shown in histological studies that rhinovirus causes little damage to the nasal mucosa. ${ }^{8}$ The pathogenesis of rhinovirus colds may be via host response, especially the activation of the parasympathetic nervous system $^{9,10}$ and the release of inflammatory mediators, such as kinins ${ }^{11,12}$ and interferon. ${ }^{13}$

In developing a placebo that matched the ZGG lozenge in astringency, there was concern that the placebo might, because of its astringency, not be entirely devoid of activity. It was, however, considered of utmost importance to have a placebo that was truly indistinguishable for the ZGG lozenge. The placebo was not devoid of activity, as demonstrated by the finding of a difference in duration between day 1 entrants and day 2 entrants who took placebo - a difference in the direction opposite to that expected.

A high standard was used to determine the mean duration of colds in the present study. In the literature quoting mean duration of a common cold, ${ }^{3,14-17}$ it has been specified that the cold is considered to have begun when one or more symptoms are present for 2 days, or two symptoms are present for 1 day. In the present study, the onset of the cold was defined as the time when the first symptom was recognized by the patient. The literature is less specific in defining the end of a cold, but the most conservative investigators consider a cold over when no more that one symptom remains, or when the patient believes that the cold is over. Employing the stated criteria in those studies ${ }^{3,14-16}$ untreated patients were symptomatic for a total of 9.2 days.

Mean durations, whether the patients were receiving ZGG or placebo, were found to be strongly dependent upon the number of days (1 or 2) that the patients had had symptoms prior to starting treatment. The mean total duration for the day 1 ZGG-treated patients was 5.3 days ( 1 day prior to treatment plus 4.3 days on treatment). This constitutes a $42 \%$ reduction in the duration of the common cold for the day 1 ZGG-treated patients. Patients treated with placebo from day 1 had colds for a mean total of 6.5 days, corresponding to a $29 \%$ reduction in duration, which shows a measurable effect of the astringent placebo used in this study.

The rate of reduction of the severity of symptoms may be interpreted as another measure of efficacy. Even with the recognition that placebo in the present study was not entirely devoid of activity, there was a significant $(P<0.025)$ reduction in the severity of symptoms in the ZGG treatment group compared with placebo at day 7; day 7 has been commonly used for comparison in other published studies that include duration and symptom severity data. ${ }^{1,2,15}$

The effects observed in the present study 
indicate that there may be a $1-2$ day 'window are coming down with a cold and are of opportunity' for treatment with ZGG before motivated to begin treatment. The present the common cold takes hold. It could be study suggests that the use of properly determined from further studies whether cold formulated zinc gluconate may indeed be a symptoms would last an even shorter time if useful approach.

ZGG treatment were begun on the day of It is hypothesized that zinc ions work in symptom onset. There were only two patients two ways: as an antiviral agent, as demontreated in each study group from day 0: the strated byin vitro studies, ${ }^{29}$ and directly on two ZGG-treated patients in this category had the trigeminal nerve as an astringent. The symptoms for a total of 3 and 4 days; and the placebo used in the present study was matched corresponding placebo-treated patients had in astringency to the ZGG lozenge; therefore, symptoms for 4 and 10 days. It is possible that it was not surprising to discover that the if treatment were started within hours of the placebo appeared to have some activity, onset of symptoms, the overall reduction in although significantly less that ZGG. symptom duration could be shortened to approximately 3.5 days, representing a $62 \%$ reduction. This is in agreement with anecdotal information obtained from casual use of the present ZGG formulation, i.e. that when the lozenges were used at an early stage, the cold seemed to be on 'fast forward'.

The literature on the common cold contains many studies on prophylactic use of such agents as interferons ${ }^{-26}$ and synthetic drugs $^{8,27,28}$ in attempts to prevent colds or ACKNOWLEDGEMENTS

shorten their duration. Interferon, for example This study was sponsored by Godfrey Science has demonstrated varying degrees of \& Design, Inc., Huntingdon Valley, prophylactic efficacy. ${ }^{22,24,26}$ Success with Pennsylvania, USA and by a grant from the such agents has proven to be elusive to Rorer Pharmaceutical Corp., Fort Washington, date, ${ }^{8,19-28}$ as most treatments either have had limited efficacy or have had side-effects, such as nasal irritation, that are more severe than the symptoms of the cold. Very few agents have been found to have any effect once the symptoms have developed, i.e. the time when cold sufferers become aware that they
The common cold causes a large economic impact upon world productivity because of the lost man hours. ${ }^{30}$ The positive finding of the present report and speculations regarding a mode of action provide a reason for hope that a safe, practical, effective, convenient and inexpensive treatment may now be at hand to alleviate substantially these burdens.
Pennsylvania, USA. We are indebted to $\mathbf{J}$ Buckley, RPh, C Bradley, RN, S MacDonald, RN, G Poinsette, MA, E Walkling, BA, and Y Baumgartner, MBA, for their valuable technical assistance. Our appreciation is extended to S McKenney and S Diaz for their assistance with study administration.

\section{REFERENCES}

1 Eby GA, Davis DR. Halcomb WW: blind studyAntimicrob Agents Chemother Reduction in duration of common colds by zinc gluconate lozenges in a double- 2 Fair BM. Conner EM, Bates RF et al. Two 
randomized controlled trials of zinc gluconate lozenge therapy of experimentally induced rhinovirus colds. Antimicrob Agents Chemothev987; 31: 1183 - 1187.

3 Douglas RM, Miles HB, Moore BLtt,al. Failure of effervescent zinc acetate lozenges to alter the course of upper respiratory tract infections in Australian adults. Antimicrob Agents Chemothd $987 ; 31$ : 1263 - 1265.

4 Smith DS, Helzner EC, Nuttall CE dt,al. Failure of zinc gluconate in treatment of acute upper respiratory tract infections. Antimicrob Agents Chemothdr989;33: $646-648$.

5 Godfrey JC: Zinc for the common cold. Antimicrob Agents Chemothdr988;32: $605-606$.

6 Zarembo JE, Godfrey JC, Godfrey NJ: Zinc(II) in saliva: determination of concentrations produced by several different formulations of zinc gluconate lozenges containing common excipients. Pharm Sci1992;81: 128-130.

7 Al-Nakib W, Higgins PG, Batstone Cf,al. Prophylaxis and treatment of rhinovirus colds with zinc gluconate lozengek. Antimicrob Chemother 987;20:893 901.

8 Gaffey MG, Hayden FG, Boyd Jet, al. Ipatropium bromide treatment of experimental rhinovirus infection. Antimicrob Agents Chemothdr988;32: 1644 - 1647.

9 Gaffey MG, Gwaltney JM Jr, Dressier WE. et al: Intranasally administered atropine methonitrate treatment of experimental rhinovirus colds Am Rev Respir Dis 987; 135: 241 - 244.

10 Gaffey MG, Gwaltney JM Jr, Sastro a, al. Intranasally and orally administered treatment of experimental rhinovirus colds. Am Rev Respir Dis 987;136:555 - 560.

11 Proud D, Naclerio RM, Gwaltney JM $\&$ t. al. Kinins are generated in nasal secretions during natural rhinovirus colds. Infect Dis 1990;161:120 - 123.

12 Proud D, Reynolds CJ, Lacapra 8t, al. Nasal provocation with bradykinin induces symptoms of rhinitis and a sore throat.Am Rev Respir Dis 988; 137:613 $-616$.

13 Chonmaitree T, Lett-Brown MA, Tsong Y, et al: Role of interferon in leukocyte histamine release caused by common respiratory viruses $I$ Infect Dis1988:157: $127-130$.

14 Gwaltney JM Jr, Hendley JO, Simon al. Rhinovirus infections in an industrial population. II. Characteristics of illness and antibody response $A M A 1967 ; 202$ : 494 - 500.

15 Karlowski TR, Chalmers TC, Frenkel LD, et al: Ascorbic acid for the common cold. JAMA 1975;231:1038 - 1042.

16 Scott GM, Phillpotts RJ, Wallace et, al. Purified interferon as protection against rhinovirus infection $B r$ Med $J 1982 ; 284$ : 1822 - 1825.

17 Gwaltney JM Jr: The common cold. In: Principles and Practice of Infectious Dis eases(Mandell GL, Douglas RG, Bennett JE, eds). New York: John Wiley, 1979; pp 429-435.

18 Curley FJ, Irwin RS, Pratter MRt, al. Cough and the common coldt.m Rev Respir Dis1988;138:305 - 311.

19 Douglas RM, Moore BW, Miles HBt,al. Prophylactic efficacy of intranasal alpha2 interferon against rhinovirus infections in a family settingN Engl $J$ Medl 986;314: $65-70$.

20 Farr BM, Gwaltney JM Jr, Adams KAr,al. Intranasal interferon-alpha-2 for prevention of natural rhinovirus colds. Antimicrob Agents Chemothdr984;26: $31-34$.

21 Greenberg SB, Harmon MW, Couch R\&B, al. Prophylactic effect of low doses of 
human leukocyte interferon against infection with rhinovirus. J Infect Dis 1982; 145: 542 - 546.

22 Hayden FG, Albrecht JK, Kaiser DLet al: Prevention of natural colds by contact prophylaxis with intranasal alpha-2 interferon. $N$ Engl J Med 1986; 314: 71 -75.

23 Hayden FG, Gwaltney JM Jr: Intranasal interferon-alpha, for prevention of rhinovirus infection and illness $J$ Infect Dis 1983; 148: 543 - 550.

24 Hayden FG, Kaiser DL, Albrecht JK: Intranasal recombinant alfa- $2 b$ interferon treatment of naturally occurring common colds. Antimicrob Agents Chemother 1988; 32: 224 - 230.

25 Herzog C, Berger R, Fernex M, et al: Intranasal interferon (rIFN-A) for contact prophylaxis against common cold: a randomized, double-blind placebocontrolled study. Antiviral Res 1986; 6: $171-176$.

26 Merigan TC, Reed SE, Hall TS, et al: Inhibition of respiratory virus infection by locally applied interferon.Lancet 1973; i: $563-567$.
27 Al-Nakib W, Higgins PG, Barrow GIęt al: Suppression of colds in human volunteers challenged with rhinovirus by a new synthetic drug (R61837). Antimicrob Agents Chemother 1989; 33: 522 - 525.

28 Hayden FG, Gwaltney JM Jr: Prophylactic activity of enviroxime against experimentally induced rhinovirus type 39 infection Antimicrob Agents Chemother 1982; 22: 1004 - 1007.

29 Merluzzi VJ, Cipriano D, McNeil Det al: Evaluation of zinc complexes on the replication of rhinovirus 2 in vitro. Res Commun Chem Pathol Pharmacol 1989; 66: 425 - 440.

30 Couch RB: The common cold: control? Infect Dis 1984; 150: 167 - 173.

J C Godfrey, B Conant Sloane, D S Smith, J H Turco, N Mercer and N J Godfrey Zinc Gluconate and the Common Cold: a Controlled Clinical Study The Journal of International Medical Research 1992; 20: 234-246

Received for publication 24 January 1992 Accepted 31 January 1992

(C) Copyright 1992 Cambridge Medical Publications Ltd

\section{Address for correspondence DR J C GODFREY}

1649 Old Welsh Road, Huntingdon Valley, Pennsylvania, PA 19006, USA. 


\begin{tabular}{|c|c|}
\hline Volume $20 \cdot$ Number 3 & $\begin{array}{l}\text { The Journal of } \\
\text { International Medical Research }\end{array}$ \\
\hline
\end{tabular}

\section{RESEARCH REPORTS}

Zinc gluconate and the common cold: a controlled clinical study

J C Godfrey, B Conant Sloans, D S Smith, J H Turco, N Mercer and $N$ J Godfrey ................................................................................. 234 